\title{
JULIAN HAPPES
}

Albert-Ludwigs-Universität Freiburg

E-Mail: julian.happes@geschichte.uni-freiburg.de

\author{
REZEPTION, TRANSFORMATION \\ UND FUNKTIONALISIERUNG DES KETZERPROZESSES \\ GEGEN JAN HUS \\ IN DER ÜBERLIEFERUNG DER KONSTANZER \\ KONZILSCHRONIK UND DER SCHWEIZER CHRONISTIK \\ DES 15. JAHRHUNDERTS
}

Der Prozess gegen Jan Hus und Hieronymus von Prag auf dem Konstanzer Konzil, der bekanntlich in der Verurteilung und Verbrennung der beiden böhmischen Gelehrten ebendort endete, zählt zu den bedeutendsten Ereignissen des Konzils. Es kann wohl als dasjenige gelten, das die Gemüter bis heute am nachhaltigsten bewegt hat und weiterhin bewegt ${ }^{1}$. Bereits kurze Zeit nach dem Konstanzer Konzil diente vor

1 Vgl. T. A. Fudge, The trial of Jan Hus: medieval heresy and criminal procedure, (2013) und J. Keǰ̆, Die Causa Johannes Hus und das Prozessrecht der Kirche, (2005); zum Konstanzer Konzil aus theologischer Sicht vgl. W. Brandmüller, Das Konzil von Konstanz, 1414-1418, 2 Bde., (Konziliengeschichte. Reihe A: Darstellungen $\left.1,{ }^{2} 1997-1999\right)$, T. M. Buck, H. Kraume, Das Konstanzer Konzil: Kirchenpolitik - Weltgeschehen - Alltagsleben, (2013); Das Konstanzer Konzil als europäisches Ereignis: Begegnungen, Medien und Rituale, hg. v. G. Signori, B. Studt, (Vorträge und Forschungen 79, 2014); eine kompakte Einführung bei A. Frenken, Das Konstanzer Konzil, (Kohlhammer-Urban-Akademie, 2015). 
allem die Person des Jan Hus der religiösen und politischen Bewegung des Hussitismus als Galions- und Märtyrerfigur ${ }^{2}$. In den folgenden Jahrhunderten blieb Jan Hus je nach Sicht des Rezipienten als Häretiker oder Reformer präsent. Selbst die moderne Forschung zu seiner Person und seinem Tod ließ immer wieder block-, national- und konfessionspolitische Tendenzen erkennen, die zu erheblichen Fehleinschätzungen in Hinblick auf seine Rolle führten ${ }^{3}$.

Schon die Haltungen der zeitgenössischen Berichterstatter des Konstanzer Konzils divergierten stark: Das Zeugnis des Peter von Mladoniowitz, der im Gefolge Hussens nach Konstanz reiste, berichtete naturgemäß positiv über den Böhmen und wurde damit zu einer entscheidenden Quelle für die anschließende Martyrisierung des Gelehrten in den böhmischen Ländern. Die Chronik des Konstanzer Konzils des Ulrich Richental entwirft hingegen ein negatives Bild des ,Erzketzers' ${ }^{4}$. Auch in der Schweizer Chronistik des 15. Jahrhunderts hielt der Prozess gegen Jan Hus Einzug, meist ausgehend von der um

2 Vgl. P. Rychterova, Die Verbrennung von Johannes Hus als europäisches Ereignis. Öffentlichkeit und Öffentlichkeiten am Vorabend der hussitischen Revolution, in: Politische Öffentlichkeit im Spätmittelalter, hg. v. M. Kintzinger, B. Schneidmüller, (Vorträge und Forschungen 75, 2011), S. 361-382; zum Hussitismus vgl. nur F. Šmahel, Die hussitische Revolution, 3 Bde., (Monumenta Germaniae Historica. Schriften 43, 2002) und Die hussitische Revolution: religiöse, politische und regionale Aspekte, hg. v. F. Machilek, (Forschungen und Quellen zur Kirchen- und Kulturgeschichte Ostdeutschlands 44, 2012).

3 Einen Überblick über den historiographischen Umgang mit der Person Jan Hus ab dem 15. Jahrhundert bis heute bei: T. A. Fudge, Jan Hus: Religious reform and social revolution in Bohemia, (International library of historical studies 73, 2010), S. 190-224; vgl. auch J. Randák, Jan Hus 600 - ein unübersichtliches Jubiläum, 2016, abrufbar im Internet: http://erinnerung.hypotheses.org/583 (besucht am 14.4.2016).

4 Zum Vergleich der beiden Darstellungen H. Herkommer, Die Geschichte vom Leiden und Sterben des Jan Hus als Ereignis und Erzählung, in: Literatur und Laienbildung im Spätmittelalter und in der Reformationszeit: Symposion Wolfenbüttel 1981, hg. v. L. Grenzmann, K. Stackmann, (Germanistische Symposien, Berichtsbände 5, 1984), S. 114-146. 
1420 entstandenen Berner Chronik Konrad Justingers und der Konstanzer Konzilschronik 5 .

Der vorliegende Beitrag setzt sich zum Ziel, die Rezeption des Husprozesses in der Überlieferung der Konstanzer Konzilschronik und der Schweizer Chronistik des 15. Jahrhunderts nachzuvollziehen. Es stellt sich die Frage, ob die Geschehnisse um Jan Hus auf dem Konstanzer Konzil in den verschiedenen Phasen der chronikalischen Überlieferung unterschiedliche Wertungen erfahren haben bzw. ob die verschiedenen Überlieferungsträger die Person des Jan Hus und dessen Verurteilung und Verbrennung im sozialen Kontext ihrer zeitlichen und räumlichen Entstehung transformieren und umfunktionalisieren. Die Erfahrung der Hussitenkriege in den Jahrzehnten nach dem Konzil könnte eine solche Neubewertung evoziert haben. Der Prozess wird in der Konstanzer Konzilschronik wie auch in der Schweizer Chronistik auf verschiedenen medialen Ebenen behandelt. Neben den chronikalischen Aufzeichnungen werden die einzelnen,Stationen' des Prozesses in Illustrationen wiedergegeben. Daher gilt es, die Abhängigkeiten bzw. Abweichungen zwischen textlicher und bildlicher Wiedergabe aufzuzeigen und unter Berücksichtigung der bisherigen Forschungen gegebenenfalls neu zu bewerten.

Dazu soll im ersten Abschnitt des Beitrags (1) die komplexe Überlieferungslage der Konstanzer Konzilschronik in gebotener Kürze vorgestellt und bewertet sowie ein theoretischer Ansatz für den Umgang mit dieser eingeführt werden. Hierbei stütze ich mich auf die umfassenden Forschungen Thomas Martin Bucks, der mit der Vorlage einer kritischen Leseausgabe der Konstanzer Konzilschronik deren Erforschung entscheidend erleichtert hat ${ }^{6}$. Gleichermaßen werden soweit

5 Vgl. F. Matouš, Johannes Hus in der Schweizer Chronistik des 15. und 16. Jahrhunderts, in: Jan Hus: zwischen Zeiten, Völkern, Konfessionen: Vorträge des internationalen Symposions in Bayreuth vom 22. bis 26. September 1993, hg. v. F. Seibt, (Veröffentlichungen des Collegium Carolinum 85, 1997), S. 367-373.

6 Chronik des Konstanzer Konzils 1414-1418 von Ulrich Richental, eing. und hg. v. T. M. Buck, (Konstanzer Geschichts- und Rechtsquellen 41, ${ }^{4} 2014$ ); zurzeit entsteht 
möglich die Abhängigkeiten der Schweizer Chroniken des 15. Jahrhunderts untereinander und zur Konstanzer Konzilschronik geklärt.

Im zweiten Abschnitt (2) sollen die Illustrationen des Husprozesses in den unterschiedlichen Überlieferungsträgern der Konstanzer Konzilschronik analysiert werden. Die heterogenen Entstehungskontexte der einzelnen illuminierten Handschriften lassen eine voneinander abweichende ,Tendenz' in den Darstellungen erwarten. In einem weiteren Schritt werden die textlichen Unterschiede der einzelnen Überlieferungsträger ausgewertet und mit den Bildinhalten verglichen. Grundlegende Vorarbeit leistete hierzu Gisela Wacker mit ihrer 2002 vorgelegten Dissertation, die anhand einer umfassenden Bildsynopse versucht, den ,Urbilderzyklus' zu ermitteln und gleichzeitig nach den Wirkungsabsichten der überlieferten Handschriften und Drucke fragt ${ }^{7}$.

Im dritten Teil (3) wird die bildliche und textliche Wiedergabe des Husprozesses in den einzelnen Schweizer Chroniken des 15. Jahrhunderts verglichen und den Darstellungen in der Konstanzer Konzilschronik gegenübergestellt. Abschließend (4) wird ausgehend von den gewonnenen Erkenntnissen kritisch zur bisherigen Forschung Stellung bezogen und ein möglicher Neuzugang im Umgang mit multimedialen historiographischen Zeugnissen des Spätmittelalters vorgestellt.

unter seiner Leitung eine kritische digitale Edition der drei ,Haupthandschriften ' der Konstanzer Konzilschronik: T. M. Buck, Monumenta Germaniae Historica-Edition der Richentalchronik des Konstanzer Konzils. Beschreibung des Vorhabens - Projektanträge, abrufbar im Internet: https://www.ph-freiburg.de/fileadmin/dateien/fakultaet3/sozialwissenschaft/geschichte/Buck/DFG-Antrag2013.pdf (besucht am 21.10.2015); mein besonderer Dank gilt Thomas Martin Buck für die Möglichkeit zur Einsichtnahme in seine ungedruckte Habilitationsschrift: T. M. Buck, Textkritische Untersuchungen zur Konzilschronik Ulrich Richentals. Auf dem Wege zu einer Neuedition, (2000).

7 G. Wacker, Ulrich Richentals Chronik des Konstanzer Konzils und ihre Funktionalisierung im 15. und 16. Jahrhundert. Aspekte zur Rekonstruktion der Urschrift und zu den Wirkungsabsichten der überlieferten Handschriften und Drucke, (2002), abrufbar im Internet: https://publikationen.uni-tuebingen.de/xmlui/handle/10900/46177 (besucht am 21.10.2015). 
1.

Die Überlieferung der Konstanzer Konzilschronik setzt ab den 1460er Jahren, also erst rund 40 Jahre nach dem vermuteten Abfassungsbeginn in den 1420er Jahren, ein ${ }^{8}$. In den zehn Jahren zwischen 1460 und 1470 entstanden elf der heute noch vorhandenen 16 handschriftlichen Überlieferungsträger. Bereits 1483 gelangte die Konstanzer Konzilschronik in Augsburg in den Druck ${ }^{9}$. Diese massive Häufung der Handschriftenproduktion und die frühe Drucklegung deuten auf ein verstärktes Interesse am Konstanzer Konzil in der Stadt und ihrer Umgebung hin. Dies korreliert mit einer auch anderenorts zu beobachtenden vermehrten Produktion volkssprachlicher historiographischer Werke, so auch im eidgenössischen Raum.

Die überlieferten Handschriften weichen in Anlage und Aufbau partiell stark voneinander ab. Eine, Urschrift', wie sie vor allem von der älteren Forschung gerne postuliert wurde, scheint es nicht gegeben zu haben. Es ist durchaus denkbar, dass die Konstanzer Konzilschronik

8 Für eine Auflistung aller vorhandenen Überlieferungsträger siehe unten Anhang 1: Handschriften und Drucke der Konstanzer Konzilschronik; zur Überlieferung: T. M. Buck, Einleitung, in: Chronik des Konstanzer Konzils, S. XIII-LIX, hier S.XXIV-XXXV; hier auch eine Auflistung der umfangreichen Veröffentlichungen Bucks zur Konstanzer Konzilschronik: S. LIVf.; vgl. auch T. M. Buck, Zur Überlieferung der Konstanzer Konzilschronik Ulrich Richentals, „Deutsches Archiv für Erforschung des Mittelalters", 66 (2010), S. 93-108 und Wacker, Funktionalisierung, S. I-XXXII.

9 Die Konstanzer Konzilschronik zählt damit zu den ersten gedruckten historiographischen Werken; A.-D. von den Brincken, Die Rezeption mittelalterlicher Historiographie durch den Inkunabeldruck, in: Geschichtsschreibung und Geschichtsbewusstsein im späten Mittelalter, hg. v. H. Patze, (Vorträge und Forschungen 31, 1987), S. 215-237, hier S. 226; vgl. M. Mertens, Früher Buchdruck und Historiographie. Zur Rezeption historiographischer Literatur im Bürgertum des deutschen Spätmittelalters beim Übergang vom Schreiben zum Drucken, in: Studien zum städtischen Bildungswesen des späten Mittelalters und der frühen Neuzeit: Bericht über Kolloquien der Kommission zur Erforschung der Kultur des Spätmittelalters, 1978 bis 1981, hg. v. B. Moeller (et. al.), (Abhandlungen der Akademie der Wissenschaften in Göttingen, Philologisch-Historische Klasse 3/137, 1983), S. 83-111. 
bereits während des Entstehungsprozesses als ,offener Text' angelegt wurde ${ }^{10}$. Die Konstanzer Konzilschronik setzt sich aus mehreren Teilen zusammen, die zwar unterschiedliche Funktionen erfüllen, aber dennoch eng miteinander verwoben sind. Neben dem chronologischen Textteil und den Illustrationen sind dies ein Teilnehmer- und Wappenverzeichnis ${ }^{11}$.

Die Überlieferung lässt sich in drei idealtypische Gruppen einteilen ${ }^{12}$ : Die sogenannte subjektive Gruppe ist aus der Erzählperspektive Richentals geschrieben, sie betont seine Rolle während des Konzils und bietet den umfangreichsten Textkorpus. Die so bezeichnete objektive Gruppe tilgt den Erzähler Richental zu Gunsten einer objektivierten Sicht; sie hebt die Rolle der Stadt und Bürgerschaft Konstanz während des Konzils hervor. Sind in den ersten beiden Gruppen die Illustrationen in den Textteil integriert und das Teilnehmer- resp. Wappenverzeichnis hintangestellt, so verändert die umfangreichste, aus Mischhandschriften bestehende Gruppe die Position der einzelnen Elemente oder tilgt diese ganz. Einige Handschriften stellen den Listenteil voran, übertragen lateinische Passagen ins Volkssprachliche, streichen einzelne Illustrationen oder lassen diese vollständig aus. Es findet eine

10 Buck, Einleitung, S. XXVIII-XXX; „offener Text“ im Verständnis der „New Philology“, vgl. G. M. Spiegel, History, Historicism, and the Social Logic of the Text in the Middle Ages, „Speculum“, 65 (1990), S. 59-86 und Philologie als Textwissenschaft. Alte und neue Horizonte, hg. v. H. Tervooren, H. Wenzel, (Zeitschrift für Deutsche Philologie, Sonderheft 116, 1997).

11 Vgl. zu den Teilnehmerlisten: J. Riegel, Die Teilnehmerlisten des Konstanzer Konzils. Ein Beitrag zur mittelalterlichen Statistik, (1913) und T. M. Buck, Und wie vil herren dar koment, sy wärind gaistlich oder sy wäremd weltlich. Zu den Namen- und Teilnehmerlisten der Konstanzer Konzilschronik Ulrich Richentals, in: Das Konstanzer Konzil als europäisches Ereignis: Begegnungen, Medien und Rituale, hg. v. G. Signori, B. Studt, (Vorträge und Forschungen 79, 2014), S. 255-304; zu den Wappenverzeichnissen vgl. zuletzt: C. Rolker, Die Richental-Chronik als Wappenbuch, „Deutsches Archiv für die Erforschung des Mittelalters", 71 (2015), S. 57-103.

12 Zur Einteilung der Handschriften siehe unten Anhang 1: Handschriften und Drucke der Konstanzer Konzilschronik. 
offensichtliche Umfunktionalisierung der Chronik zu Gunsten eines neuen Nutzungszusammenhanges statt ${ }^{13}$.

Maßgeblich beteiligt an der Rezeption war der Konstanzer Bürger und Chronist Gebhard Dacher, der sich für die umfassendsten und intensivsten redaktionellen Umarbeitungen verantwortlich zeigt ${ }^{14}$. Es kann vermutet werden, dass er auch die frühe Drucklegung beförderte bzw. die Vorlage für den Druck schuf ${ }^{15}$.

Bereits kurz nach dem mutmaßlichen Entstehungszeitraum der Konstanzer Konzilschronik wurde diese durch die Schweizer Chronistik rezipiert ${ }^{16}$. So enthält die Winterthurer Handschrift eigentlich keine Version der Konstanzer Konzilschronik, sondern die Berner Chronik Konrad Justingers, die in Teilen der Konstanzer Konzilschronik folgt ${ }^{17}$. Die in der zweiten Hälfte des 15. Jahrhunderts in Bern entstandenen Bilderchroniken übernehmen die chronikalischen Darstellungen Justingers bis 1420. Es sind dies die bebilderten Werke Diebold Schillings und Bendicht Tschachtlans ${ }^{18}$. Auch die Zürcher Chroniken schöpfen unter anderem

13 Buck, Einleitung, S. XXXVIIf.

14 Er gilt auch als Redakteur der sogenannten Konstanzer Chronik: Die „Konstanzer Chronik" Gebhart Dachers. „By des Byschoffs zyten volgiengen disz nachgeschriben ding vnd sachen... "Codex Sangallensis 646: Edition und Kommentar, hg. v. S. Wolff, (Konstanzer Geschichts- und Rechtsquellen 40, 2008).

15 Buck, Einleitung, S. XIX.

16 Zur Schweizer Chronistik des 15. Jahrhunderts allgemein, J. P. Bodmer, Chroniken und Chronisten im Spätmittelalter (Monographien zur Schweizer Geschichte 10, 1976).

17 Vgl. Wacker, Funktionalisierung, S. XVII und K. Jost, Konrad Justinger (ca. 1365-1438): Chronist und Finanzmann in Berns großer Zeit, (Vorträge und Forschungen, Sonderband 56, 2011), S. 29f., 419; zu Konrad Justinger vgl. Ebd. und H. Strahm, Der Chronist Konrad Justinger und seine Berner Chronik von 1420, (Schriften der Berner Burgerbibliothek, 1978); ediert bei: Die Berner-Chronik des Conrad Justinger nebst vier Beilagen, hg. v. G. Studer, (1871), abrufbar im Internet: http://www.digibern.ch/katalog/ berner-chronik-conrad-justinger (besucht am 21.10.2015).

18 Vgl. C. G. Baumann, Über die Entstehung der ältesten Schweizer Bilderchroniken (1468-1485). Unter besonderer Berücksichtigung der Illustrationen in Diebold Schillings Großer Burgunderchronik in Zürich, (1971) und R. Schmid-Keeling, Die Stadtansichten in den Schweizer Bilderchroniken, in: Schweizer Städtebilder. Urbane 
aus der Konstanzer Konzilschronik ${ }^{19}$, gleiches gilt für die sogenannte Klingenberger Chronik des Rapperswiler Stadtschreibers Eberhard Wüst ${ }^{20}$. Für einen intensiven Austausch zwischen dem Bodensee- und dem schweizerischen Raum stehen vier Codices, die neben Auszügen aus der Konstanzer Konzilschronik auch Schweizer Chroniken enthalten. In diesen Handschriften sind die Illustrationen bewusst ausgelassen und reagieren damit auf ein verändertes Rezipientenbedürfnis ${ }^{21}$. In den Jahrzehnten nach dem Konzil fand also ein umfassender Austausch von chronikalischem Wissen zwischen dem schweizerischen und dem Bodenseeraum statt. Es kann davon ausgegangen werden, dass sich dieser Austausch mit dem Verlust des Thurgaus von Konstanz an die Eidgenossenschaft 1460 intensivierte ${ }^{22}$.

Ikonographien (15.-20. Jahrhundert), hg. v. B. Roeck (et al.), (2013), S. 37-49; die Chroniken Diebold Schillings sind ediert bei: Die Berner Chronik des Diebold Schilling 1468-1484, hg. v. G. Tobler, 1-2 (1897-1901); Faksimiles samt Textedition und Kommentar: Die große Burgunderchronik Diebold Schillings von Bern, Faksimileausgabe, hg. v. A. A. Schmid, (1958), Kommentarband, hg. v. dems., E. Bürgisser, (1985) und Diebold Schillings Spiezer Bilderchronik, Faksimile der Bilderhandschrift Mss. $h$. $h$. I. 16 der Burgerbibliothek Bern, hg. v. H. Haeberli, (1990), Kommentarband, hg. v. dems., V. Bartlome, U. M. Zahnd, (1990); Die Schweiz im Mittelalter in Diebold Schillings Spiezer Bilderchronik Studienausgabe zur Faksimile-Edition der Handschrift Mss. h. h. I. 16 der Burgerbibliothek Bern samt Textedition, hg. v. Dens., (1990); die Tschachtlanchronik samt Textedition und Kommentar: Tschachtlans Bilderchronik: die älteste Schweizer Bilderchronik, Zentralbibliothek Zürich Ms. A 120, hg. v. V. Bartlome, A. A. Schmid, (1986), Kommentarband, hg. v. Dens., (1988).

19 Vgl. R. Gamper, Die Zürcher Stadtchroniken und ihre Ausbreitung in die Ostschweiz. Forschungstexte, Überlieferung, Analyse der Chroniktexte, (Mitteilungen der Antiquarischen Gesellschaft in Zürich, 52/2, 1984); die Zürcher Chroniken sind ediert bei: Chronik der Stadt Zürich. Mit Fortsetzungen, hg. v. J. Dierauer, (Quellen zur Schweizer Geschichte 18, 1900).

20 Edition bei: Die sog. Klingenberger Chronik des Eberhard Wuest, Stadtschreiber von Rapperswil, hg. v. B. Stettler, (Mitteilungen zur vaterländischen Geschichte 53, 2007).

21 Die Handschriften St. Gallen [Sg], Innsbruck [I] sowie die beiden in Zürich verwahrten $\left[\mathrm{Z}_{1}, \mathrm{Z}_{2}\right]$; vgl. Wacker, Funktionalisierung, $\mathrm{S}$. XIVf., XVIIf. und Buck, Einleitung, S. XLf.

22 Vgl. W. Matthiessen, Ulrich Richentals Chronik des Konstanzer Konzils. Studien 
Die bisherige Forschung zur Konstanzer Konzilschronik stellte neben den Illustrationen meist die Abhängigkeiten der überlieferten Handschriften und Drucke zueinander ins Schlaglicht. Auch wenn die Arbeiten Gisela Wackers und Wilhelm Matthiessens wichtige Vorarbeiten zum Verständnis der Funktion einzelner Überlieferungsträger leisteten, so erfolgte dies größtenteils auf Basis der älteren Edition bzw. des Faksimiles der Konstanzer Handschrift ${ }^{23}$.

Fragt man jedoch nicht mehr nur nach der Intention des möglichen Autors bzw. Auftraggebers, sondern darüber hinaus nach den Refunktionalisierungen der einzelnen Überlieferungsträger in späteren Gebrauchssituationen, so müssen diese konsequent unter Berücksichtigung des jeweiligen sozialen Kontextes und des möglichen Rezipientenkreises untersucht und bewertet werden. Nur so lassen sich sichere Erkenntnisse über die Rezeption, Transformation und Nutzung einzelner Themenbereiche gewinnen ${ }^{24}$.

zur Behandlung eines universalen Großereignisses durch die bürgerliche Chronistik, „Annuarium Historiae Conciliorum“, 17 (1985), S. 71-191, 323-455, hier S. 413f.; zum Bodenseeraum als Kontaktzone vgl. H. Maurer, Schweizer und Schwaben: ihre Begegnung und ihr Auseinanderleben am Bodensee im Spätmittelalter, (Konstanzer Universitätsreden 136, ${ }^{2}$ 1991) und ders., Vom Konzil bis zum Beginn des 16. Jahrhunderts, (Konstanz im Mittelalter 2, 1989), S. 48-195.

23 Auf Basis der Aulendorfer Handschrift: Ulrichs von Richental Chronik des Konstanzer Concils. 1414 bis 1418, hg. v. M. R. Buck, (Bibliothek des litterarischen Vereins in Stuttgart 163, 1882); Faksimile der Konstanzer Handschrift samt Transkription: Das Konzil zu Konstanz MCDXIV-MCDXVIII von Ulrich Richental. Faksimile der Chronik des Konstanzer Rosgartenmuseums, ca. 1465; Kommentarband, hg. v. O. Feger, (1964).

24 Vgl. J. Happes, Transformation und Nutzung der Konstanzer Konzilschronik im späten 15. Jahrhundert, „Mitteilungen der Residenzen-Kommission der Akademie der Wissenschaften zu Göttingen. Stadt und Hof ", 4 (2015). P. Eckhart, B. Studt, Das Konzil im Gedächtnis der Stadt. Die Verhandlung von Wissen über die Vergangenheit in der städtischen Geschichtsschreibung am Oberrhein im 15. und 16. Jahrhundert, in: Urbanität. Formen der Inszenierung in Texten, Karten, Bildern, hg. v. U. Schneider, M. Stercken, (Städteforschung. Reihe A: Darstellungen 90, 2016), S. 83-104. 


\section{2.}

In den vorhandenen sieben illustrierten Handschriften der Konstanzer Konzilschronik und den drei Drucken finden sich insgesamt fünf Darstellungen, die den Fall Hus thematisieren ${ }^{25}$. Nur die Aulendorfer Handschrift überliefert alle Abbildungen. In den anderen Handschriften fehlen einzelne Illustrationen; sie wurden von den Malern nicht mehr ausgeführt, später entfernt oder waren gar nicht vorgesehen ${ }^{26}$. Die teilweise stark dezimierten Bilderzyklen lassen daher kaum allgemeingültige Schlussfolgerungen darüber $\mathrm{zu}$, wie diese ursprünglich angelegt gewesen sein könnten. Es kann jedoch vermutet werden, dass die Stellung von Bild und Text, wie sie in der Aulendorfer, der Prager und der Wiener Handschrift ausgeführt ist, der Ursprungssituation am nächsten kommt ${ }^{27}$.

Gisela Wacker konnte anhand eines breit aufgestellten Vergleichs der Illustrationen der Konstanzer Konzilschronik mit anderen zeitgenössischen bebilderten Chroniken und möglichen Vorbildern plausibel machen, dass die Maler sich an vorhandenen Schemata und Similes orientierten, um der Themenvielfalt Herr zu werden ${ }^{28}$. Dabei wurde

25 Siehe unten Anhang 2: Husdarstellungen in den illustrierten Handschriften und Drucken der Konstanzer Konzilschronik; ein vollständiges vergleichendes Bilderverzeichnis bei: T. M. Buck, Figuren, Bilder, Illustrationen. Zur piktoralen Literalität der Richental-Chronik, in: Scientia Veritatis. Festschrift für Hubert Mordek zum 65. Geburtstag, hg. v. O. Münch, T. Zotz, (2004), S. 411-444, hier S. 438-443 und Wacker, Funktionalisierung, Anhang 1; für eine aktuelle Beschreibung der illustrierten Handschriften vgl. Katalog der deutschsprachigen illustrierten Handschriften des Mittelalters 3, hg. v. N. H. Ott (et. al.), (2011), S. 450-487; die im Folgenden herangezogenen Illustrationen sind sämtlich digitalisiert und online frei zugänglich. Die im Text verwendete Nummerierung der Illustrationen ist in Anhang 2 aufgeschlüsselt, die Links zu den Digitalisaten finden sich in Anhang 3: Abbildungsnachweise.

26 Buck, Illustrationen, S. 416.

27 Ebd., S. 419.

28 Wacker, Funktionalisierung, S. 8; vgl. N. H. Ott, Zum Ausstattungsanspruch illustrierter Städtechroniken. Sigismund Meisterlin und die Schweizer Chronistik als Beispiele, in: Poesis et pictura: Studien zum Verhältnis von Text und Bild in Handschriften und 
meist ein biblisches Simile mit einem profanen Inhalt überblendet, die „formale Angleichung des historischen Geschehens an einen Prototyp" bildete damit zugleich die Voraussetzung für das inhaltliche Verständnis des Rezipienten ${ }^{29}$. Gleiches gilt auch für die Jan Hus und Hieronymus von Prag betreffenden Bilder. Diese lehnen sich an gängige spätmittelalterliche Passionsdarstellungen an ${ }^{30}: 1$. Verhaftung des Jan Hus (Abb. $1^{31}$ ): Die nur in der Aulendorfer und Prager Handschrift vorhandene Darstellung orientiert sich an der Vorführung Jesu vor Repräsentanten der weltlichen und geistlichen Macht. Die überkreuzten Hände sollen hier als ein Symbol für die bevorstehende Erfüllung der Passion verstanden werden. 2. Degradierung des Jan Hus (Abb. 2, 5, 8): Jan Hus wird in ein priesterliches Gewand gekleidet, das ihm dann wieder abgenommen wird. Die zentralsymmetrische Haltung, der verklärte Gesichtsausdruck und der geneigte Kopf verweisen auf den ganzfigurigen Schmerzensmann. 3. Jan Hus wird zum Tode geführt (Abb. 3, 6, 8): Diese Abbildung folgt ikonographisch der Kreuztragungsszene. 4. Die Verbrennung des Jan Hus (Abb. 4, 6, 9, 10): Hier erkennt Gisela Wacker eine Parallele zur Kreuzigung Jesu, genauer gesagt zur Darstellung des volkreichen Kalvarienbergs ${ }^{32}$. Der aufrechte Balken im Rücken des Delinquenten soll symbolhaft für das Kreuz stehen. Die Bewaffneten erinnern an römische Soldaten. Die Aufschrift, Heresiarchia' auf der Inful Hussens verweist wie das INRI auf dem Kreuz auf den Grund der Strafe. 5. Die Verladung der Asche (Abb. 7, 9, 10): Das letzte Bild des Huszyklus zeigt die Entsorgung der Asche des Verbrannten im Rhein.

alten Drucken; Festschrift für Dieter Wuttke zum 60. Geburtstag, hg. v. S. Füssel, J. Knape, (Saecula spiritalia, Sonderband, 1989), S. 77-106.

29 Wacker, Funktionalisierung, S. 119.

30 Das Folgende nach Ebd., S. 121-130.

31 Siehe Anm. 25.

32 Das Motiv des volkreichen Kalvarienbergs entwickelte sich im 14. Jahrhundert in Italien und war im 15. Jahrhundert vorherrschend in der Kreuzigungsikonographie: Wacker, Funktionalisierung, S. 126; vgl. E. Roth, Der volkreiche Kalvarienberg in Literatur und Bildkunst des Spätmittelalters, (Philologische Studien und Quellen 2, ${ }^{2} 1967$ ). 
Dies hatte zum Zweck, eine Verwendung der Überreste als Reliquien zu verhindern. Gisela Wacker sieht in der kreuzförmigen Deichsel des Karrens eine diskrete Anspielung auf die Legende der Kreuzauffindung, die als ironischer Hinweis auf die vergeblichen Bemühungen der Henker, die Martyrisierung und Heiligenverehrung Hussens zu verhindern, verstanden werden kann.

In Anlehnung an die ersten Forschungen Rudolf Kautzschs zu den Abhängigkeiten der Handschriften untereinander auf Basis einer kunstgeschichtlichen Analyse der Abbildungen ${ }^{33}$ versucht Gisela Wacker die Handschriften auf verschiedene Vorstufen zurückzubeziehen: Die Handschriften Prag und Aulendorf würden auf einer Vorlage aus der Mitte der 1420er Jahre basieren, die Wiener und Konstanzer über Zwischenstufen auf einer Handschrift, die vor 1431 entstanden sein müsste. Die St. Georgener Handschrift und die von ihr abhängigen Drucke ließen sich ebenfalls auf diese Version zurückführen. Die St. Petersburger Handschrift kombiniere diese Version mit einer weiteren um 1435 entstandenen. Letztere Handschrift sei eine reine Bilderhandschrift, die ausschließlich mit lateinischen Tituli auskomme ${ }^{34}$. Damit käme sie der vermuteten Ausgangssituation sehr nahe ${ }^{35}$. Nach Gisela Wackers Analyse würden die heutigen volkssprachlichen Handschriften auf einer lateinischen Quellensammlung samt angehängtem Bilderzyklus beruhen. Die eigentliche volkssprachliche Chronik wäre somit bereits eine erste Bearbeitungsstufe, die noch von Ulrich Richental redigiert worden wäre ${ }^{36}$.

Die zwischen 1424 und 1435 entstandenen verschiedenen Versionen, die Gisela Wacker als Vorlagen der heute noch vorhandenen illustrierten Handschriften vermutet, würden demnach auf die Be-

33 Vgl. R. Kautzsch, Die Handschriften von Ulrich Richentals Chronik des Konstanzer Konzils, „Zeitschrift für die Geschichte des Oberrheins“, 9 (1894), S. 443-496, Tafel XVII-XXI.

34 Vgl. Wacker, Funktionalisierung, S. 18-46.

35 Ebd., S. 90.

36 Ebd., S. 120. 
dürfnisse der Rezipienten in den 1420er und 1430er Jahren reagieren; die den Passionsdarstellungen folgenden Husillustrationen ließen nach ihrer Schlussfolgerung eine prohussitische Haltung des Autors Ulrich Richental erkennen, die dieser je nach Rezipientenbedürfnis entschärfte ${ }^{37}$. Vergleicht man stellvertretend die Illustrationen der Prager und der Wiener Handschrift miteinander, so zeigen sich minimale Abweichungen zwischen den einzelnen Bildszenen. So kommt die Wiener Handschrift beispielsweise ohne Wappen und Herrschaftszeichen aus, wohingegen die Prager Handschrift die Büttel der Stadt Konstanz durch das Konstanzer Stadtwappen kennzeichnet (Abb. 1, 3), die an der Degradierung beteiligten geistlichen Würdenträger durch ihre Wappen identifiziert (Abb. 2) und Kurfürst Ludwig von der Pfalz durch seine Standarte ausweist (Abb. 3, 4). Gisela Wacker sieht in der Prager Handschrift eine bewusste Dämonisierung Hussens, die die eigentliche prohussitische Grundhaltung der Vorlage quasi ins Gegenteil verkehrt ${ }^{38}$. Daraus folgernd vermutet sie für diese Handschrift einen reformkritischen Käufer aus dem Landadel oder dem aufstrebenden Bürgertum ${ }^{39}$. Ähnliche Argumente findet sie für die übrigen illustrierten Handschriften: die prohussitisch-reformorientierte Ausrichtung der Konstanzer Handschrift ließe einen patrizischen Käuferkreis erwarten, das Gleiche gelte für die Wiener Handschrift. Die Aulendorfer Handschrift betone die Passion Hussens, kritisiere aber gleichzeitig die Prunksucht der römischen Kurie. Gisela Wacker hält einen Käufer aus dem stadtgeistlichen Milieu für wahrscheinlich ${ }^{40}$.

Die Prager und die St. Georgener Handschrift, letztere fungierte wahrscheinlich als Vorlage für die Drucke und wurde möglicherweise zu diesem Zwecke angefertigt, sind beide unter der Ägide Gebhard Dachers erstellt worden. Die St. Georgener Handschrift ist stark ver-

37 Wacker, Funktionalisierung, S. 209-222.

38 Ebd., S. 229.

39 Ebd., S. 231.

40 Ebd., S. 228-248. 
stümmelt und die Abbildungen zum Husprozess daher nicht mehr vorhanden, allerdings können diese über die Drucke rekonstruiert werden $^{41}$. Gisela Wacker sieht in den Abbildungen der Drucke eine Betonung der Passion Hussens und schließt daraus auf eine Kehrtwende Gebhard Dachers von einer antihussitischen Haltung in der Prager Handschrift zu einer prohussitischen in den Drucken ${ }^{42}$.

Die Abbildungen des Husprozesses folgen, wie Gisela Wacker richtig erkannt hat, spätmittelalterlichen Passionsdarstellungen. Gleichzeitig verwenden einzelne Abbildungen Similes, die aus einem anderen Kontext stammen könnten. So finden sich in jeder Verbrennungsdarstellung zu Seiten des Delinquenten Personen, die mit Mistgabeln auf diesen einwirken (Abb. 4, 6, 9, 10). Dies könnte eine Anlehnung an die Teufelsfiguren in spätmittelalterlichen Weltgerichtsdarstellungen sein, die die Verdammten und Ketzer mit Gabeln oder Spießen in den Höllenschlund stoßen und Hus damit eindeutig als zu dieser Gruppe gehörig ausweisen ${ }^{43}$.

Die Illustratoren der Konstanzer Konzilschronik verwenden zwar tradierte Vorbilder zur Wiedergabe neuer Inhalte, jedoch nimmt die,tatsächliche Wahrheit‘ einen höheren Stellenwert ein als „die Anbindung der eigenen Gegenwart an die Wahrheit des heilsgeschichtlichen Modells“44. Damit ist nicht ausgeschlossen, dass der zeitgenössische Betrachter die verwendeten Similes erkannte, jedoch halte ich es für fragwürdig, ob dies eine Deutung der Hinrichtung Hussens im Sinne eines Martyriums zur Folge hatte. Ebenso könnte die Auslegung antithetisch erfolgen: Die Degradierung Hussens steht im Gegensatz zur Einkleidung eines Priesters,

41 Dass die Abbildungen vorhanden gewesen sein müssen, beweist ein Hinweis in der Handschrift auf diese, G, fol. 21v; zur Vorlage der Handschrift St. Georgen für den Erstdruck vgl. Buck, Einleitung, S. XIX; vgl. dagegen Wacker, Funktionalisierung, S. 255.

42 Ebd., S. 257f.

43 Diesen Hinweis verdanke ich Hr. Pfr. Jörg Hirsch; vgl. D. Urbach, Weltgericht und städtische Selbstdarstellung. Das Wandgemälde am Triumphbogen des Ulmer Münsters, (2001), S. 136-141 und H. Vorgrimler, Die Geschichte der Hölle, (1994), S. 214-233.

44 Ott, Ausstattungsanspruch, S. 87. 
die papierene Inful ist an eine Bischofsmitra angelehnt und spricht Jan Hus damit als,Bischof aller Ketzer, als Erzketzer an ${ }^{45}$.

Die Unterschiede zwischen den Abbildungen der einzelnen Handschriften sind meines Erachtens nicht signifikant genug, um konkrete thematische Richtungswechsel zu verorten, auch wenn anhand einzelner wiederkehrender Details durchaus auf gemeinsame Vorlagen geschlossen werden kann. Die unterschiedliche Qualität und Maltechnik der verschiedenen Bilderzyklen und nicht zuletzt die ,künstlerische Freiheit', die die einzelnen Illustratoren gegenüber ihren Vorlagen bewahrt haben könnten und die nicht klar benennbar ist, lassen damit keine konkreten Erkenntnisse über die thematische Ausrichtung der Handschriften oder der möglichen Vorlagen zu. Damit bleiben Aussagen über die Meinungen und Haltungen des Autors oder identifizierbarer Bearbeiter Spekulation.

Vergleicht man die Erzählung des Husprozesses auf textlicher Ebene, so zeigen sich Unterschiede sowohl im Inhalt als auch in der Anlage der Erzählung. Die Handschriften Prag und Aulendorf, die der subjektiven Gruppe zugerechnet werden, erzählen die Ereignisse knapper als die Handschriften Konstanz und Wien, die der objektiven Gruppe angehören. Der Überschuss an Informationen besteht aus Texteinschüben, die einen Rechtfertigungszweck erkennen lassen. Beide Handschriftengruppen bringen bereits in Kapitel 109 und 110 die Erzählung der angeblichen Flucht Hussens ${ }^{46}$. Dieser Einschub rechtfertigt die Verhaftung Hussens, durch seine Flucht bricht er das ihm von König Sigismund zugesicherte freie Geleit. Damit wird dieser entlastet ${ }^{47}$. Die Handschriften Konstanz und Wien bieten hier weitere

45 Vgl. M. Kubíková, The heretic's Cap of Jan Hus, „Bohemian Reformation and Religious Praxis", 4 (2002), S. 143-150.

46 Chronik des Konstanzer Konzils, S. 43f.; A foll. 90f., Pr fol. 32v, K fol. 39v, W fol. 62v; die Fluchtgeschichte wird im Hauptteil der Erzählung nochmals wiedergegeben.

47 Vgl. R. Hoke, Der Prozeß gegen Jan Hus und das Geleit König Sigmunds. Ein Beitrag zur Frage nach der Kläger- und Angeklagtenrolle im Konstanzer Prozeß von 1414/1415, „Annuarium Historiae Conciliorum“, 15 (1983), S. 172-193. 
Informationen, die das Verhalten König Sigismunds zusätzlich legitimieren. Gleichzeitig wird mehrfach betont, dass Jan Hus von gelert lüt in der götlichen kunst überwunden ward und des bösen globen abgestanden sin. Zwar erwähnen auch Prag und Aulendorf die gelert herren in theologia, allerdings erwähnen sie nicht, dass Hus in einem gelehrten Gespräch widerlegt worden sei.

Die eigentliche Erzählung über Prozess, Verurteilung und Verbrennung Hussens folgt in den Kapiteln 147-156 der Konstanzer Konzilschronik $^{48}$. Wieder zeigt ein Vergleich der Passagen einige Überschüsse in den Handschriften Konstanz und Wien: Der vorgebliche Disput Hussens mit Gelehrten wird wieder stärker betont. Die weiteren Abweichungen sind von keiner thematischen Relevanz.

Die Handschrift St. Georgen und die ihr folgenden Drucke weichen von der Erzählfolge der ersten beiden Handschriftengruppen ab. So werden aus der gesamten Erzählung sieben kleinere Einzelgeschichten herausgenommen; neben anderen Indizien spricht das für einen veränderten Leser- und Buchmarkt, auf den der Redaktor Gebhard Dacher reagiert ${ }^{49}$. Die Huserzählung steht an letzter Stelle in der Handschrift St. Georgen und entspricht inhaltlich weitestgehend der Wiedergabe in den Handschriften Konstanz und Wien ${ }^{50}$. Die Stellung am Ende des Chroniktextes spräche nach Gisela Wacker für eine positive Deutung Hussens durch Gebhard Dacher ${ }^{51}$. Die exponierte, aus dem Text herausgelöste Stellung der Huserzählung am Ende der Handschrift St. Georgen verweist zwar auf ein besonderes, möglicherweise gestiegenes Leserinteresse, eine positive Deutung Hussens durch den Redaktor Gebhard Dacher lässt sich jedoch nicht zwingend schlussfolgern.

48 Chronik des Konstanzer Konzils, S. 61-66; A foll. 128-134, Pr foll. 44r-47v, K foll. $54 \mathrm{v}-57 \mathrm{r}, \mathrm{W}$ foll. $77 \mathrm{v}-81 \mathrm{r}$.

49 Buck, Einleitung, S. XXX, XXXVII.

50 Die 7. Texteinheit ist durch Textverlust unvollständig, kann aber nach $\mathrm{D}_{1}$ ergänzt werden: G foll. 267r-268v, $D_{1}$ foll. 244r-245v, G foll. Br-Cr.

51 Wacker, Funktionalisierung, S. 253. 
In der Texteinheit, die derjenigen entspräche, in der die Huserzählung in den übrigen Handschriften eingebettet ist, bringt die Handschrift St. Georgen ausschließlich eine knappe Zusammenfassung sowie ein Verweis auf die nachgetragene ausführliche Wiedergabe der Geschehnisse $^{52}$. Die ausgegliederte Fluchtgeschichte fehlt, wird aber wie in den anderen Handschriften im eigentlichen Erzählteil wiederholt.

Der Vergleich der textlichen Wiedergabe des Husprozesses lässt in den einzelnen Überlieferungsträgern einen entscheidenden Unterschied erkennen: Die Handschriften, die der objektiven Gruppe zuzuordnen sind, bieten einen Textüberschuss, der eine legitimatorische Funktion besitzt: Der Bruch des freien Geleits wird gerechtfertigt, der Irrglaube Hussens und seiner Anhänger betont. Das fügt sich in das Gesamtbild der Handschrift Konstanz ein: Diese bietet gegenüber der subjektiven Gruppe inhaltliche Überschüsse, die die Rolle und Bedeutung der Stadt Konstanz während des Konzils hervorheben. Es wird eine harmonische, geordnete Gesamtsituation beschworen, die die Rechtmäßigkeit des Konzils und damit auch des Husprozesses betonen soll $^{53}$. Das Bedürfnis nach einer, legitimatorischen Absicherung' über die Rechtmäßigkeit des Husprozesses und der daraus resultierenden Verbrennung des Böhmen beweisen die hinzugefügten Textstellen. Doch lässt der Textvergleich keine gesicherten Aussagen darüber zu, ob einzelne Überlieferungsträger damit eine positivere Haltung gegenüber Jan Hus vermitteln wollen.

Vergleicht man den Text und die Illustrationen der einzelnen Handschriften miteinander, so zeigt sich, dass die Bilder nicht in allen Details den Vorgaben des Textes folgen bzw. partiell von diesen offensichtlich abweichen. So betont der Chroniktext beispielsweise, dass bei der Hinführung zur Richtstätte die Hände Hussens nicht gefesselt waren. Jedoch zeigen die Illustrationen in der Wiener Handschrift Hus mit

$52 \mathrm{G}$ foll. 21r-21v.

53 Vgl. Buck, Einleitung, S. XXIV-XXVI, XXXVII und Matthiessen, Universales Großereignis, S. 369-394. 
gebundenen Händen (Abb. 6). Gisela Wacker sieht die gefesselten Hände in der Tradition der Kreuztragungsikonographie ${ }^{54}$. Sie deutet diese und andere Abweichungen als Hinweis darauf, dass der Autor Richental seine latent prohussitische Haltung im Text, abgesehen von subtilen Anspielungen, hinter der Verwendung von gängigen Ketzertopoi verbergen musste, wohingegen die Bildebene eine, offenere ' Kritik an der causa fidei zuließ ${ }^{55}$. Dieser Schluss fußt auf einem neuzeitlichen Verständnis von einer, realitätsnahen' Wiedergabe im Text, die sich in den Bildern wiederfände. Eine vollständige Übereinstimmung zwischen bildlicher und textlicher Ebene ist allerdings kaum erwartbar. Der oder die Maler sind nicht identisch mit dem oder den Schreiber/n und dem Redaktor. Wer welchen Einfluss ausübte und ob sie alle dieselben Ziele verfolgten, lässt sich für uns nicht mehr eindeutig feststellen.

Text und Bild erfüllen trotz ihrer gemeinsamen Anlage unterschiedliche Funktionen. Thomas Cramer unterscheidet zwischen der Ereignisgeschichte des Textes und der exemplarischen Geschichte der Bilder. In den Handschriften Aulendorf, Konstanz und Wien, sind die Illustrationen nicht willkürlich über den Text gestreut, sondern zu episodischen Bildfolgen gebündelt, innerhalb derer sich die Bilder zu eigenen Erzählzusammenhängen fügen ${ }^{56}$. Die Existenz von reinen

54 Wacker, S. $123 \mathrm{f}$.

55 Ebd., S. 129-132.

56 T. Cramer, Bilder erzählen Geschichte. Die Illustrationen in Ulrich Richentals Chronik als Erzählung in der Erzählung, in: Erzählungen in Erzählungen. Phänomene der Narration in Mittelalter und Früher Neuzeit, hg. v. H. Haferland, M. Mecklenburg, (Forschungen zur Geschichte der älteren deutschen Literatur 19, 1996), S. 327-349, hier S. 332, 337; Andrea Löther und Gerrit Jasper Schenk konnten anhand der abgebildeten Prozessionen und Einzüge in der Konstanzer Konzilschronik ebenfalls nachweisen, dass Text und Bild unterschiedliche Informationsschwerpunkte setzen und sich damit in ihrer Funktionalität unterscheiden, vgl. A. Löther, Rituale im Bild. Prozessionsdarstellungen bei Albrecht Dürer, Gentile Bellini und in der Konzilschronik Ulrich Richentals, in: Mundus in imagine: Bildersprache und Lebenswelten im Mittelalter: Festgabe für Klaus Schreiner, hg. v. ders., (1996), S. 99-123 und G. J. Schenk, Die Lesbarkeit von Zeichen der Macht und die Grenzen der Macht von Zeichen auf dem Konstanzer Konzil am Beispiel des Einzugs Papst Johannes' XXIII. (1414), in: Das Konstanzer Konzil als europäisches 
Bildhandschriften und unillustrierten Überlieferungsträgern zeigt, dass sowohl die Abbildungen als auch der Chroniktext als eigenständige Erzählungen funktionieren konnten.

3.

Das Konstanzer Konzil wird bereits kurze Zeit nach dem Ereignis durch die Schweizer Chronistik rezipiert. Die Berner Chronik des Konrad Justinger schöpft dabei aus der Konzilschronik, eine persönliche Anwesenheit des Berners auf dem Konstanzer Konzil ist wahrscheinlich. Die das Konzil betreffenden Teile sind dabei stark gekürzt und umgearbeitet. Der Schwerpunkt liegt auf Ereignissen, an denen die Berner Bürgerschaft direkt beteiligt war ${ }^{57}$. Die Partizipation der Schweizer Städte an der militärischen Auseinandersetzung zwischen König Sigismund und Herzog Friedrich von Österreich nimmt daher den breitesten Raum $e^{5}{ }^{58}$. Dies gilt auch für die Zürcher und die Klingenberger Chronik. Diese übernimmt den das Konstanzer Konzil betreffenden Teil aus den Zürcher Chroniken und ergänzt ihn durch zusätzliche Informati-

Ereignis: Begegnungen, Medien und Rituale, hg. v. B. Studt, G. Signori, (Vorträge und Forschungen 79, 2014), S. 255-304.

57 Die Berner-Chronik des Konrad Justinger, Kap. 345-423, S. 209-243; vgl. Matthiessen, Universales Großereignis, S. 404-408; Katrin Jost bestreitet meines Erachtens zu Unrecht eine Nutzung der Konstanzer Konzilschronik durch Konrad Justinger. Sie begründet diese Annahme mit dem späten Einsetzen der Überlieferung nach dem Tod Justingers. Allerdings ist es sehr wahrscheinlich, dass zwischen dem Entstehungszeitraum der Konstanzer Konzilschronik und der ältesten heute noch erhaltenen Handschrift andere Abschriften existierten, die der Berner Chronist verwendet haben könnte; vgl. Jost, Konrad Justinger, S. 230-232; vgl. auch H. Strahm, Der Chronist Konrad Justinger, S. 65-82.

58 Zur Auseinandersetzung vgl. W. Baum, Die Habsburger in den Vorlanden 1386-1486. Krise und Höhepunkt der habsburgischen Machtstellung in Schwaben am Ausgang des Mittelalters, (1993), S. 109-168 und B. Stettler, Die Eidgenossenschaft im 15. Jahrhundert. Die Suche nach einem gemeinsamen Nenner, (2004), S. 123-138. 
onen aus einer Handschrift des Konstanzer Konzils ${ }^{59}$. Die Chroniken Diebold Schillings und Bendicht Tschachtlans orientieren sich an der Chronik Konrad Justingers, der Schwerpunkt verbleibt auf der Auseinandersetzung zwischen König Sigismund und Herzog Friedrich von Österreich bzw. der Rolle Berns in diesem Konflikt. Es finden keine nennenswerten inhaltlichen Veränderungen statt ${ }^{60}$. Die zur Verfügung stehenden Inhalte werden also im Hinblick auf das Funktionsbedürfnis selektiert und in den Chroniktext eingearbeitet.

Der Prozess gegen Jan Hus und Hieronymus von Prag wird in der Schweizer Chronistik tradiert, obwohl bei diesem Themenkomplex kein konkreter Bezug zu den Schweizer Städten besteht. In der Chronik Konrad Justingers wird die Geschichte dann auch massiv gekürzt ${ }^{61}$. Der Textvergleich zeigt, dass die Chroniken Diebold Schillings und Bendicht Tschachtlans keinerlei Veränderungen vornehmen ${ }^{62}$. Die überlieferten Handschriften der Zürcher Chroniken erwähnen den Prozess gegen Jan Hus nicht, lediglich die erste Fortsetzung enthält zwei äußerst kurze Passagen zu den Hussitenkreuzzügen ${ }^{63}$. Die Chroniken belassen es also meist bei einer kurzen Erwähnung, weisen einige Ungenauigkeiten auf und die Ereignisse erfahren keine weiterführende Interpretation $^{64}$.

59 Chronik der Stadt Zürich, Kap. 191-195, S. 176-182; Klingenberger Chronik, fol. 87-105, S. 174-191; vgl. Matthiessen, Universales Großereignis, S. 413f. und Gamper, Zürcher Stadtchroniken, S. 3f.

60 Vgl. Matthiessen, Universales Großereignis, S. 410-412.

61 Die Berner-Chronik des Konrad Justinger, Kap. 414, S. 238.

62 Diebold Schillings Spiezer Bilderchronik, Kap. 473, S. 558; Diebold Schillings Amtl. Berner Chronik, Kap. 473, S. 367f., Bendicht Tschachtlans Berner Chronik, Kap. 413, S. 267.

63 Es handelt sich um die Teilnahme von Zürchern an einem Hussitenkreuzzug und um die Bereitstellung von Hellebarden: Erste Zürcher Fortsetzung, fol. 56, 58, S. 187f., S. 192.

$64 \mathrm{Zu}$ diesem Ergebnis kommt auch František Matouš, Johannes Hus in den Schweizer Chroniken. 
In der Amtlichen Berner und der Spiezer Chronik des Diebold Schilling wird der Husprozess auch auf bildlicher Ebene wiedergegeben. Die Bilderchronik Tschachtlans enthält keine Illustrationen hierzu. Beide Chroniken beschränken sich auf den zentralen Vorgang der Verbrennung, eine Anlehnung an die Abbildungen der Konstanzer Konzilschronik ist wahrscheinlich. Die Verbrennungsdarstellung in der Amtlichen Berner Chronik bietet im Vergleich zu denen in den Handschriften des Konstanzer Konzils einen anderen Bildaufbau und liefert zusätzliche Informationen (Abb. 11): Die Verbrennung hat noch nicht begonnen, sondern ist noch in der Vorbereitung begriffen. Die damit beschäftigten Personen sind durch die umgegürteten Schwerter als Stadtbüttel erkennbar, im Hintergrund befinden sich links die weltlichen Würdenträger angeführt von König Sigismund, rechts die Repräsentanten des geistlichen Standes mit dem Papst an der Spitze. Im Zentrum des Bildes harrt Jan Hus, hier ohne Inful, seiner Verbrennung, die ihn umgebenden Bücher symbolisieren die gleichzeitige Vernichtung seiner Irrlehren. Einen Hinweis auf Konstanz als Ort des Geschehens findet sich nicht.

Im Gegensatz hierzu wird in der Spiezer Chronik die Stadt Konstanz klar benannt (Abb. 12); sie ist im Hintergrund dargestellt und durch das Stadtwappen gekennzeichnet. Die Personen vor dem Stadttor sind durch ihre Hüte als städtische Würdenträger erkennbar. Der Scheiterhaufen brennt bereits. Nach Gisela Wacker folgt die Amtliche Berner Chronik dem christologischen Modell der Konstanzer Konzilschronik, die Spiezer Chronik nicht ${ }^{65}$. In ihrer stilistischen Gesamtheit ist die Spiezer Chronik der Konstanzer Konzilschronik jedoch näher ${ }^{66}$.

Die beiden Darstellungen weisen in den Details deutliche Unterschiede auf, die in Ausführung und Anlage auf einen geänderten

65 Wacker, Funktionalisierung, S. 141.

66 Vgl. L. E. Saurma-Jeltsch, Die Illustrationen und ihr stilistisches Umfeld, in: Diebold Schillings Spiezer Bilderchronik, Kommentar zur Faksimileausgabe der Handschrift Mss. h.h.I.16 der Burgerbibliothek Bern, hg. v. H. Haeberli, C. v. Steiger, (1990), S. 31-71, hier S. 52-58. 
Rezipientenkreis schließen lassen: Die Amtliche Berner Chronik entstand im Auftrag der Stadt, die Spiezer Chronik für den Berner AltSchultheißen Rudolf von Erlach. Entsprechend finden sich im Textteil vereinzelt Hervorhebungen dieser Person bzw. seiner Familie. Auch der Bilderzyklus unterscheidet sich von der Amtlichen Berner Chronik in seiner Ausführung ${ }^{67}$. Es erfolgt allerdings keine grundsätzliche inhaltliche Umarbeitung. Die Abbildungen der Husverbrennung wirken im Vergleich zu denen der Konstanzer Konzilschronik, komprimiert', das Gleiche gilt für den Text. Das Ereignis besitzt für den Berner Rezipientenkreis der 1480er Jahre keine akute Relevanz, es findet daher keine Aktualisierung oder Umfunktionalisierung statt. Eine seriöse Aussage über die Haltung der Rezipienten, des Malers oder Diebold Schillings gegenüber der causa Hus ist nicht möglich.

Eine Sonderstellung nimmt hier die sogenannte Klingenberger Chronik ein. Diese fügt an die Erzählung des Husprozesses als dessen direkte Folge den Aufstand der Hussiten in Böhmen und Prag und die daraus resultierenden Hussitenkreuzzüge an. Als Vorlage dient ihr hierbei die Chronik des Eberhard Windecke ${ }^{68}$. Die Politik König Sigismunds gegenüber den Hussiten findet hier im Gegensatz zur Chronik Windeckes deutliche Kritik ${ }^{69}$. Die Chronik verfolgt damit ein konkret fassbares Ziel: Die Hussiten werden mit den Appenzellern und Schwytzern gleichgesetzt und unter der Bezeichnung puren vereint; das Reich, der Adel und das Haus Habsburg werden als Ordnungsmacht den abweichenden Kräften gegenübergestellt. Die Handschrift wirft König Sigismund die Paktierung mit den puren in Schwaben und mit den ungelöbigen in Böhmen vor ${ }^{70}$. Hier findet also eine fassbare

67 Vgl. Saurma-Jeltsch, Illustrationen, S. 42-45.

68 Stettler, Einleitung, in: Die sog. Klingenberger Chronik, S. 22 f.

69 Vgl. B. Studt, Zwischen Kurfürsten, Kurie und Konzil. Die Hussitenpolitik König Sigismunds, in: Sigismund von Luxemburg: ein Kaiser in Europa: Tagungsband des internationalen historischen und kunsthistorischen Kongresses in Luxemburg, 8.-10. Juni 2005, hg. v. M. Pauli, F. Reinert, (2006), S. 13-23.

70 Stettler, Einleitung, S. 15f, 20. 
Umfunktionalisierung und Aktualisierung des Husprozesses statt, er dient in Kombination mit den anschließenden Hussitenzügen der Rechtfertigung des Vorgehens der Herzöge von Österreich gegen die Appenzeller in den sogenannten Appenzellerkriegen ${ }^{71}$.

4.

Der Prozess gegen Jan Hus wurde sowohl in den verschiedenen Überlieferungsträgern der Konstanzer Konzilschronik als auch in der Schweizer Chronistik des 15. Jahrhunderts rezipiert. Wie die Untersuchung jedoch zeigen konnte, muss diese Rezeption nicht zwangsläufig mit einer Transformation bzw. Umfunktionalisierung des Themas im sozialen Kontext der Entstehung und Rezeption der einzelnen Handschriften einhergehen. Die Handschriften der Konstanzer Konzilschronik weichen in wenigen Details ihrer bildlichen und mehr noch textlichen Wiedergabe des Ereignisses voneinander ab. Aufgrund der partiellen Unvollständigkeit der Illustrationszyklen, deren Gründe heute nur noch schwer zu benennen sind, sowie der Unklarheiten in Hinblick auf die Beteiligung verschiedener Maler, Einflussnahme des Redaktors, Abhängigkeit von Vorbildern etc. können kaum gesicherte Erkenntnisse über die Haltungen einzelner Beteiligter bzw. Rezipienten zu Jan Hus getroffen werden. Die textlichen Unterschiede der Handschriften offenbaren eine offensichtlich legitimatorische Funktion, die allerdings im zeitlichen Rahmen der 1420er und 1430er Jahre bewertet werden sollte. Die Hussitenpolitik König Sigismunds während dieser Zeit war umstritten und damit auch seine Rolle bei der Verurteilung und Verbrennung Hussens. Im Jahrzehnt zwischen 1460 und 1470 waren die Hussiten kein Thema der Tagespolitik mehr. Die Handschriften des Konstanzer Konzils, die während dieser Zeit entstanden sind,

71 Vgl. nur Die Appenzellerkriege - eine Krisenzeit am Bodensee, hg. v. P. Niederhäuser, (Forschungen zur Geschichte des Vorarlbergs 7, 2006). 
lassen daher auch keine Neuinterpretation oder Instrumentalisierung der causa Hus erwarten.

Ein vergleichender Blick in die Konstanzer Chronik, die in diesem Zeitraum von Gebhard Dacher erstellt worden ist, unterstützt diesen Eindruck: Nur an zwei Stellen wird knapp auf die Hussiten verwiesen ${ }^{72}$. Auch die rein geographische Distanz zu den militärischen Auseinandersetzungen in Böhmen spricht gegen eine aktuelle Brisanz des Themas in Konstanz bereits ab den 1420er und mehr noch ab den 1460er Jahren $^{73}$.

Der Schweizer Chronistik kann Ähnliches attestiert werden: Die Wiedergabe des Konstanzer Konzils wird in Hinblick auf die Beteiligung der Schweizer Städte stark selektiert, der Husprozess erfährt eine entsprechend umfassende Kürzung. Eine Ausnahme ist die sogenannte Klingenberger Chronik: Hier findet eine bewusste Umfunktionalisierung des Ereignisses statt: Nicht mehr die Bedeutung des Husprozesses für das Konstanzer Konzil, sondern für die folgenden Ereignisse wird betont, die ketzerischen Kräfte der Hussiten mit den Appenzellern und Schwytzern gleichgesetzt.

Im Zuge der Reformation erhält die Person des Jan Hus und die Umstände seines Todes neue Brisanz. Die Chroniken eines Aegidius Tschudi, Johannes Cochläus oder Johannes Stumpf nähern sich dem Konstanzer Konzil und der causa Hus zielgerichtet: Die vorhandenen Informationen werden selektiert, die Ereignisse neu interpretiert und im zeitlichen und räumlichen Kontext umfunktionalisiert ${ }^{74}$. Eine be-

72 Beteiligung von Konstanzern am Hussitenfeldzug und Erwähnung der Eroberung der Prager Neustadt durch die Utraquisten, Die „Konstanzer Chronik“, fol. 104ra, S. 463; fol. $142 \mathrm{rb}$, S. 535.

73 In Chroniken des nord- und ostdeutschen Raumes nehmen die Hussiten entsprechend eine deutlich prominentere Stellung ein, vgl. C. Hruschka, Kriegsführung und Geschichtsschreibung im Spätmittelalter: eine Untersuchung zur Chronistik der Konzilszeit, (Kollektive Einstellungen und sozialer Wandel im Mittelalter, n.F. 5, 2001), S. $112-142$.

74 Vgl. B. Stettler, Einleitung, in: Aegidius Tschudi. Chronicum Helveticum, 8. Teil, hg. v. Dems, (Quellen zur Schweizer Geschichte. I. Abteilung: Chroniken VII/8, 1990), 
sondere Form der Aktualisierung findet sich in einer Inkunabel des Zweitdruckes der Konstanzer Konzilschronik: Hier wurde Jan Hus von späterer Hand ein Bart gemalt, eine Anpassung an die seit dem Beginn des 16. Jahrhunderts gebräuchlichere ikonographische Darstellung des Böhmen (Abb. 15, 16).

Der Prozess gegen Jan Hus ist ein zentrales Ereignis des Konstanzer Konzils und wird entsprechend sowohl von den unterschiedlichen Überlieferungsträgern der Konstanzer Konzilschronik als auch von der Schweizer Chronistik des 15. Jahrhunderts wiedergegeben. Diese Rezeption fußt aber eben nicht auf der fortwährenden politischen oder religiösen Brisanz des Husprozesses in der zweiten Hälfte des 15. Jahrhunderts, sondern auf dessen Funktion als integraler Bestandteil einer Erzählung über das Konstanzer Konzil. Nach dieser Erkenntnis müssen gerade multimediale Handschriften historiographischen Inhalts konsequent unter der Berücksichtigung ihres sozialen Kontextes bewertet werden. Besonders bei einem historiographischen Werk wie der Konstanzer Konzilschronik, deren Überlieferung stark divergent ist, muss bei der Frage nach der konkreten thematischen Ausrichtung einzelner Handschriften Vorsicht walten, sofern Entstehungskontext, Rezipientenkreis und Abhängigkeiten nicht gänzlich zu klären sind.

S. 12-22; P. Haberkern, ,After me There Will Come Braver Men': Jan Hus and Reformation Polemics in the 1530s, „German History“, 27 (2009), S. 177-195 und P. Eckhart, Konzil und Konzilschronik im lokalen Gedächtnis. Die Kirchenversammlung in der Konstanzer Publizistik und Historiographie der Reformationszeit, in: Über die ganze Erde erging der Ruf von Konstanz. Rahmenbedingungen und Rezeption des Konstanzer Konzils, hg. v. K.-H. Braun, (Veröffentlichungen der Kommission für geschichtliche Landeskunde Baden-Württemberg) [in Vorbereitung]. 


\section{ANHANG 1:}

HANDSCHRIFTEN UND DRUCKE DER KONSTANZER KONZILSCHRONIK ${ }^{75}$

1. (Subjektive) Gruppe

$A=$ New York, New York Public Library, Spencer Collection, Nr. 32 (ehemals Aulendorf), um 1460 [illustriert]

Pr= Prag, Národní Knihovna Ceské Republiky (Cod.XVI A 17), 1464 [illustriert]

2. (Objektive) Gruppe

$\mathrm{K}=$ Konstanz, Rosgartenmuseum Konstanz (Inv. Hs. 1), um 1465 [illustriert]

$\mathrm{Pt}=$ Prag, Národní Knihovna Ceské Republiky (Cod. VII A 18) (ehemals St. Petersburg), um 1470 [illustriert]

W= Wien, Österreichische Nationalbibliothek (Cod. 3044), um 1470 [illustriert]

3. Gruppe: Mischhandschriften

$\mathrm{E}=$ Karlsruhe, BLB Karlsruhe (Cod. Ettenheim-Münster 11), um 1500 [illustriert]

$\mathrm{G}=$ Karlsruhe, BLB Karlsruhe (Cod. St. Georgen 63), um 1470 [illustriert]

$\mathrm{St}_{1}=$ Stuttgart, WLB Stuttgart, HB V 22, 1467-1469

Wo $=$ Wolfenbüttel, Herzog August Bibliothek, Cod. Guelf. 61 Aug. $2^{\circ}$, Anfang 16. Jh.

$\mathrm{St}_{2}=$ Stuttgart, WLB Stuttgart, Cod. theol. et philos. $2^{\circ} 76$, Bd. 37, Ende 17. Jh.

$\mathrm{Sg}=$ St. Gallen, Stiftsbibliothek (Cod. Germ 657), 1470-1480

$\mathrm{I}=\quad$ Innsbruck, Ferdinandeum, Sammlung Di Pauli 873, um 1460

$\mathrm{Z}_{1}=$ Zürich, Zentralbibliothek (Ms A 172), um 1500

$Z_{2}=$ Zürich, Zentralbibliothek (Ms A 80), um 1475

$\mathrm{Wi}=$ Winterthur, Stadtbibliothek (Perin Aa 1, foll.276-291), Mitte 15. Jh.

$\mathrm{Li}=$ Lindau, Stadtarchiv-Stadtbibliothek, P I 2, 16./17. Jh.

4. Drucke

$\mathrm{D}_{1}=$ Anton Sorg, Augsburg 1483 (Hain ${ }^{*} 5610$ ) [illustriert]

$\mathrm{D}_{2}=$ Heinrich Steyner, Augsburg 1536 [illustriert]

$\mathrm{D}_{3}=$ Siegmund Feyerabend, Frankfurt a.M. 1575 [illustriert]

75 Nach: Buck, Einleitung, S. LVIIf. 
ANHANG 2:

HUSDARSTELLUNGEN IN DEN ILLUSTRIERTEN HANDSCHRIFTEN UND DRUCKEN DER KONSTANZER KONZILSCHRONIK ${ }^{76}$

\begin{tabular}{|l|l|l|l|l|l|l|l|l|}
\hline & Pr & W & Pt & A & K & $D_{1}$ & $D_{2}$ & $\mathrm{D}_{3}$ \\
\hline $\begin{array}{l}28 \\
\text { Hus verhaftet }\end{array}$ & $\begin{array}{l}122 \mathrm{v} \\
\text { Abb. } 1\end{array}$ & & & $\begin{array}{l}135 \\
\text { ohne }\end{array}$ & & & & \\
\hline $\begin{array}{l}29 \\
\text { Hus degradiert }\end{array}$ & $\begin{array}{l}123 \mathrm{r} \\
\text { Abb. } 2\end{array}$ & $\begin{array}{l}81 \mathrm{r} \\
\text { Abb. } 5\end{array}$ & $\begin{array}{l}11 \mathrm{r} \\
\text { Abb. } 8\end{array}$ & $\begin{array}{l}136 \\
\text { ohne }\end{array}$ & $\begin{array}{l}57 \mathrm{v} \\
\text { ohne }\end{array}$ & $\begin{array}{l}33 \mathrm{v} \\
\text { Abb. 13 }\end{array}$ & $\begin{array}{l}25 \mathrm{v} \\
\text { Abb. 15 }\end{array}$ & $\begin{array}{l}22 \mathrm{v} \\
\text { Abb. } 17\end{array}$ \\
\hline $\begin{array}{l}30 \\
\text { Hus zum Tode geführt }\end{array}$ & $\begin{array}{l}123 \mathrm{v} \\
\text { Abb. } 3\end{array}$ & $\begin{array}{l}81 \mathrm{v} \\
\text { Abb. } 6\end{array}$ & $\begin{array}{l}11 \mathrm{r} \\
\text { Abb. } 8\end{array}$ & $\begin{array}{l}137 \\
\text { ohne }\end{array}$ & $\begin{array}{l}57 \mathrm{v} \\
\text { ohne }\end{array}$ & $\begin{array}{l}33 \mathrm{v} \\
\text { Abb. 13 }\end{array}$ & $\begin{array}{l}25 \mathrm{v} \\
\text { Abb. 15 }\end{array}$ & $\begin{array}{l}22 \mathrm{v} \\
\text { Abb. } 17\end{array}$ \\
\hline $\begin{array}{l}31 \\
\text { Hus verbrannt }\end{array}$ & $\begin{array}{l}124 \mathrm{r} \\
\text { Abb. } 4\end{array}$ & $\begin{array}{l}81 \mathrm{v} \\
\text { Abb. } 6\end{array}$ & $\begin{array}{l}11 \mathrm{v} \\
\text { Abb. } 9\end{array}$ & $\begin{array}{l}138 \\
\text { Abb. } 10\end{array}$ & $\begin{array}{l}58 \mathrm{r} \\
\text { ohne }\end{array}$ & $\begin{array}{l}34 \mathrm{r} \\
\text { Abb. 14 }\end{array}$ & $\begin{array}{l}26 \mathrm{r} \\
\text { Abb. 16 }\end{array}$ & \\
\hline $\begin{array}{l}32 \\
\text { Asche wird verladen }\end{array}$ & ausgef. & $\begin{array}{l}82 \mathrm{r} \\
\text { Abb. } 7\end{array}$ & $\begin{array}{l}11 \mathrm{v} \\
\text { Abb. } 9\end{array}$ & $\begin{array}{l}139 \\
\text { Abb. } 10\end{array}$ & $\begin{array}{l}58 \mathrm{r} \\
\text { ohne }\end{array}$ & $\begin{array}{l}34 \mathrm{r} \\
\text { Abb. 14 }\end{array}$ & $\begin{array}{l}26 \mathrm{r} \\
\text { Abb. } 16\end{array}$ & \\
\hline
\end{tabular}

ANHANG 3:

\section{ABBILDUNGSNACHWEISE}

- Abb. 1-4: Pr: Prag, Národní Knihovna Ceské Republiky (Cod. XVI A 17), 1464, fol. 122v, 123r, 123v, 124 r. Digitalisat im Internet abrufbar: http://v2.manuscriptorium. com/apps/main/en/index.php?request=show_tei_digidoc\&docId=set 200808 08_106_98 (besucht am 5.5.2016).

- Abb. 5-7: W: Wien, Österreichische Nationalbibliothek (Cod. 3044), um 1470, fol. 81r, 81v, 82r (S. 167-169). Digitalisat im Internet abrufbar: http://search.obvsg.at/ primo_library/libweb/action/dlDisplay.do?institution=ONB\&vid=ONB\&onCam pus=false\&lang=ger\&docId=ONB_aleph_onb06000161455 (besucht am 5.5.2016).

- Abb. 8-9: Pt: Prag, Národní Knihovna Ceské Republiky (Cod. VII A 18) (ehemals St. Petersburg), um 1470, fol. 11r, 11v. Digitalisat im Internet abrufbar: http://v2. manuscriptorium.com/apps/main/en/index.php?request=show_tei_digidoc\&doc $\mathrm{Id}=$ set04020657 (besucht am 5.5.2016).

- Abb. 10: A: New York, New York Public Library, Spencer Collection, Nr. 32 (ehemals Aulendorf), um 1460, fol. 138, 139. Einzelne Abbildungen im Internet abrufbar: http://images.nypl.org/index.php?id=427456\&t=w (besucht am 5.5.2016).

76 Nach: Buck, Illustrationen, S. 439. 
- Abb. 11: Diebold Schilling, Amtl. Berner Chronik, Bd. 1 (Bern, Burgerbibliothek, Mss.h.h.I.1), 1478-1483, S. 367. Digitalisat im Internet abrufbar: http://www.e-cod ices.unifr.ch/de/bbb/Mss-hh-I0001/367 (besucht am 5.5.2016).

- Abb. 12: Diebold Schilling, Spiezer Chronik (Bern, Burgerbibliothek, Mss.h.h.I.16), 1484-1485, S. 660. Digitalisat im Internet abrufbar: http://www.e-codices.unifr. ch/de/bbb/Mss-hh-I0016/660/0 (besucht am 5.5.2016).

- Abb. 13-14: D 1 : Anton Sorg, Augsburg 1483, (Hain * 5610), (Karlsruhe, BLB Dh9), fol. 33v, 34r (S. 66f.). Digitalisat im Internet abrufbar: http://tudigit.ulb.tu-darm stadt.de/show/inc-iii-55/0066?sid=c0f9b0a8910d947553e10f2ba566efc8 (besucht am 5.5.2016).

- Abb. 15-16: D2: Heinrich Steyner, Augsburg 1536 (München, BSB Res/2 J.pract. 169\#Beibd.4; Projektnummer: VD16 R 2202), fol. 25v, 26r (S. 50f.) Digitalisat im Internet abrufbar: http://dfg-viewer.de/show/?tx_dlf[page]=50\&tx_dlf[id]=http \%3A\%2F\%2Fdaten.digitale-sammlungen.de\%2F db\%2Fmets\%2Fbsb00074421_ mets.xml\&tx_dlf[double]=0\&cHash=8b1feca49a6e6beaa40eb69409677676 (besucht am 5.5.2016).

- Abb. 17: $D_{3}$ : Siegmund Feyerabend, Frankfurt a.M. 1575 (München; BSB Res/2 Eur. 86\#Beibd.1; Projektnummer: VD16 R 2203), fol.22v (S. 56). Digitalisat im Internet abrufbar: http://dfg-viewer.de/show/?tx_dlf[page]=56\&tx_dlf[id]=http $\% 3 \mathrm{~A}$ \%2F\%2Fdaten.digitale-sammlungen.de $\% 2 \mathrm{~F} \sim \mathrm{db} \% 2 \mathrm{Fmets} \% 2 \mathrm{Fbsb} 00054503$ mets. xml\&tx_dlf[double] $=0 \&$ cHash=ba0aaa3acec354dcabfef77e69f66ec6 (besucht am 5.5.2016).

\title{
RECEPCJA, TRANSFORMACJA, FUNKCJONALIZACJA PROCESU O HEREZJĘ PRZECIWKO JANOWI HUSOWI
}

\author{
W PRZEKAZACH KRONIKARSKICH SOBORU W KONSTANCJI ORAZ \\ KRONIKARSTWIE SZWAJCARSKIM XV WIEKU
}

\section{(STRESZCZENIE)}

Skazanie i spalenie Jana Husa oraz Hieronima z Pragi zaliczają się do najważniejszych wydarzeń soboru w Konstancji. Hus już w krótkim czasie stał się postacią martyrologiczną husytyzmu. Przez wieki był postrzegany w zbiorowej pamięci i dyskutowany w zależności od potrzeb odbiorcy jako heretyk, męczennik lub bohater narodowy. Poniższy artykuł ma na celu zbadanie recepcji, transformacji i funkcjonalizacji procesu przeciwko Janowi Husowi w świetle kroniki soboru w Konstancji oraz szwajcarskiego kronikarstwa XV wieku. Niezależnie od dotychczasowych badań analiza ta 
może udowodnić, że informacje o procesie Jana Husa stały się wprawdzie integralną częścią kroniki soboru w Konstancji i szwajcarskiego kronikarstwa XV wieku, jednakże transformacja tego wydarzenia w sensie zmiany jego znaczenia nastąpiła jedynie w pojedynczych przypadkach.

Tłumaczenie

Renata Skowrońska

\section{REZEPTION, TRANSFORMATION UND FUNKTIONALISIERUNG DES KETZERPROZESSES GEGEN JAN HUS}

IN DER ÜBERLIEFERUNG DER KONSTANZER KONZILSCHRONIK UND

DER SCHWEIZER CHRONISTIK DES 15. JAHRHUNDERTS

\section{(ZUSAMMENFASSUNG)}

Die Verurteilung und Verbrennung Jan Hussens und Hieronymus von Prags zählt zu den wichtigsten Ereignissen des Konstanzer Konzils. Hus wurde bereits kurze Zeit später zur Märtyrer- und Galionsfigur des Hussitismus. Die Person Jan Hus blieb je nach Bedürfnis des Rezipienten als Häretiker, Märtyrer oder Nationalheld im Gedächtnis der Jahrhunderte präsent und wurde kontrovers diskutiert. Der folgende Beitrag setzt sich zum Ziel die Rezeption, Transformation und Funktionalisierung des Prozesses gegen Jan Hus in der vielgestaltigen Überlieferung der Konstanzer Konzilschronik und der Schweizer Chronistik des 15. Jahrhunderts zu untersuchen. Die Untersuchung kann in Abgrenzung zur bisherigen Forschung nachweisen, dass der Prozess gegen Jan Hus zwar als integraler Bestandteil der Konstanzer Konzilschronik tradiert wird, eine Transformation und Umfunktionalisierung des Ereignisses im Sinne einer Umdeutung jedoch nur in Einzelfällen stattfindet.

\section{RECEPTION, TRANSFORMATION AND FUNCTIONALIZATION OF THE TRIAL AGAINST JOHN HUS}

IN THE MANUSCRIPT TRADITION OF THE CHRONICLE OF THE COUNCIL OF CONSTANCE AND THE SWISS HISTORIOGRAPHY IN THE 15 CENTURY

(SUMMARY)

The condemnation and burning of John Hus and Hieronymus of Prague is one of the most important events at the Council of Constance. Soon afterwards John Hus became the martyr and figurehead for the Hussites movement. According to the needs of the 
recipients, in the following centuries the person of John Hus remained in the collective memory as a heretic, martyr or national hero and was controversially discussed throughout time. The following article proposes the analysis of the reception, transformation and functionalization of the trial against John Hus within the diverse traditions of the Chronicle of the Council of Constance and the Swiss Historiography of the $15^{\text {th }}$ century. The analysis will prove in clear distinction to previous studies that the trial against John Hus was admittedly passed on as an integral part of the Chronicle of the Council of Constance, but just in isolated incidents it occurred as a transformation and refunctionalization of the event in terms of a substantial reinterpretation.

\section{Słowa kluczowe / Schlagworte / Keywords}

- Jan Hus, kronikarstwo szwajcarskie, kronika soboru w Konstancji (Ulrich Richental)

- Jan Hus, Schweizer Chronistik, Chronik des Konstanzer Konzils (Ulrich Richental)

- John Hus, swiss historiography, Chronicle of the Council of Constance (Ulrich Richental)

\section{BIBLIOGRAFIA / BIBLIOGRAFIE / BIBLIOGRAPHY}

\section{ŹRÓDłA DRUKOWANE / GEDRUCKTE QUELLEN / PRINTED SOURCES}

Chronik des Konstanzer Konzils 1414-1418 von Ulrich Richental, eing. und hg. v. T. M. Buck, (Konstanzer Geschichts- und Rechtsquellen 41, ${ }^{42014) .}$

Das Konzil zu Konstanz MCDXIV-MCDXVIII von Ulrich Richental. Faksimile der Chronik des Konstanzer Rosgartenmuseums, ca. 1465, hg. v. O. Feger, (1964); Kommentarband, hg. v. dems., (1964).

Ulrichs von Richental Chronik des Konstanzer Concils. 1414 bis 1418, hg. v. M. R. Buck, (Bibliothek des litterarischen Vereins in Stuttgart 163, 1882). 
Die „Konstanzer Chronik“ Gebhart Dachers. „By des Byschoffs zyten volgiengen disz nachgeschriben ding vnd sachen... "Codex Sangallensis 646: Edition und Kommentar, hg. v. S. Wolff, (Konstanzer Geschichts- und Rechtsquellen 40, 2008).

Die sog. Klingenberger Chronik des Eberhard Wuest, Stadtschreiber von Rapperswil, hg. v. B. Stettler, (Mitteilungen zur vaterländischen Geschichte 53, 2007).

Die Schweiz im Mittelalter in Diebold Schillings Spiezer Bilderchronik. Studienausgabe zur Faksimile-Edition der Handschrift Mss. h. h. I. 16 der Burgerbibliothek Bern samt Textedition, hg. v. H. Haeberli, V. Bartlome, U. M. Zahnd, (1990).

Diebold Schillings Spiezer Bilderchronik, Faksimile der Bilderhandschrift Mss. h. h. I. 1) der Burgerbibliothek Bern, hg. v. H. Haeberli, (1990); Kommentarband, hg. v. dems., C. v. Steiger, (1990).

Die große Burgunderchronik Diebold Schillings von Bern, Faksimileausgabe, hg. v. A. A. Schmid, (1958); Kommentarband, hg. v. dems., E. Bürgisser, (1985).

Die Berner Chronik des Diebold Schilling 1468-1484, hg. v. G. Tobler,1-2 (1897-1901). Tschachtlans Bilderchronik: die älteste Schweizer Bilderchronik, Zentralbibliothek Zürich Ms. A 120, hg. v. V. Bartlome, A. A. Schmid, (1986); Kommentarband, hg. v. dems., (1988).

Chronik der Stadt Zürich. Mit Fortsetzungen, hg. v. J. Dierauer, (Quellen zur Schweizer Geschichte 18, 1900).

Die Berner-Chronik des Conrad Justinger nebst vier Beilagen, hg. v. G. Studer, (1871). Aegidius Tschudi. Chronicum Helveticum, 8. Teil, hg. v. B. Stettler, (Quellen zur Schweizer Geschichte. I. Abteilung: Chroniken VII/8, 1990).

\section{LITERATURA / LITERATUR / LITERATURE}

Baum W., Die Habsburger in den Vorlanden 1386-1486. Krise und Höhepunkt der habsburgischen Machtstellung in Schwaben am Ausgang des Mittelalters, (1993).

Baumann C. G., Über die Entstehung der ältesten Schweizer Bilderchroniken (1468-1485). Unter besonderer Berücksichtigung der Illustrationen in Diebold Schillings Großer Burgunderchronik in Zürich, (1971).

Bodmer J. P., Chroniken und Chronisten im Spätmittelalter, (Monographien zur Schweizer Geschichte 10, 1976).

Brandmüller W., Das Konzil von Konstanz, 1414-1418, 2 Bde. (Konziliengeschichte. Reihe A: Darstellungen 1, $\left.{ }^{2} 1997-1999\right)$.

Brincken A.-D. von den, Die Rezeption mittelalterlicher Historiographie durch den Inkunabeldruck, in: Geschichtsschreibung und Geschichtsbewusstsein im späten Mittelalter, hg. v. H. Patze, (Vorträge und Forschungen 31, 1987), S. 215-237.

Buck T. M., Und wie vil herren dar koment, sy wärind gaistlich oder sy wäremd welt- 
lich. Zu den Namen- und Teilnehmerlisten der Konstanzer Konzilschronik Ulrich Richentals, in: Das Konstanzer Konzil als europäisches Ereignis: Begegnungen, Medien und Rituale, hg. v. G. Signori, B. Studt, (Vorträge und Forschungen 79, 2014), S. 255-304.

Buck T. M., Einleitung, in: Chronik des Konstanzer Konzils 1414-1418 von Ulrich Richental, eing. und hg. v. dems., (Konstanzer Geschichts- und Rechtsquellen 41, $\left.{ }^{4} 2014\right)$, S. XIII-LIX.

Buck T. M., Monumenta Germaniae Historica. Edition der Richentalchronik des Konstanzer Konzils. Beschreibung des Vorhabens - Projektanträge, abrufbar im Internet: https://www.ph-freiburg.de/fileadmin/dateien/fakultaet3/sozialwissenschaft/geschichte/Buck/DFG-Antrag2013.pdf (besucht am 21.10.2015).

Buck T. M., H. Kraume, Das Konstanzer Konzil: Kirchenpolitik - Weltgeschehen Alltagsleben, (2013).

Buck T. M., Zur Überlieferung der Konstanzer Konzilschronik Ulrich Richentals, „Deutsches Archiv für Erforschung des Mittelalters“, 66 (2010), S. 93-108.

Buck T. M., Figuren, Bilder, Illustrationen. Zur piktoralen Literalität der RichentalChronik, in: Scientia Veritatis. Festschrift für Hubert Mordek zum 65. Geburtstag, hg. v. O. Münch, T. Zotz, (2004), S. 411-444.

Buck T. M., Textkritische Untersuchungen zur Konzilschronik Ulrich Richentals. Auf dem Wege zu einer Neuedition, (2000).

Cramer T., Bilder erzählen Geschichte. Die Illustrationen in Ulrich Richentals Chronik als Erzählung in der Erzählung, in: Erzählungen in Erzählungen. Phänomene der Narration in Mittelalter und Früher Neuzeit, hg. v. H. Haferland, M. Mecklenburg, (Forschungen zur Geschichte der älteren deutschen Literatur 19, 1996), S. 327-349.

Eckhart P., Konzil und Konzilschronik im lokalen Gedächtnis. Die Kirchenversammlung in der Konstanzer Publizistik und Historiographie der Reformationszeit, in: Über die ganze Erde erging der Ruf von Konstanz. Rahmenbedingungen und Rezeption des Konstanzer Konzils, hg. v. K.-H. Braun, (Veröffentlichungen der Kommission für geschichtliche Landeskunde Baden-Württemberg) [in Vorbereitung].

Eckhart P., Studt B., Das Konzil im Gedächtnis der Stadt. Die Verhandlung von Wissen über die Vergangenheit in der städtischen Geschichtsschreibung am Oberrhein im 15. und 16. Jahrhundert, in: Urbanität. Formen der Inszenierung in Texten, Karten, Bildern, hg. v. U. Schneider, M. Stercken, (Städteforschung. Reihe A: Darstellungen 90, 2016), S. 83-104.

Frenken A., Das Konstanzer Konzil, (Kohlhammer-Urban-Akademie, 2015).

Fudge T. A., The trial of Jan Hus: medieval heresy and criminal procedure, (2013).

Fudge T. A., Jan Hus: Religious reform and social revolution in Bohemia, (International library of historical studies 73, 2010). 
Gamper R., Die Zürcher Stadtchroniken und ihre Ausbreitung in die Ostschweiz. Forschungstexte, Überlieferung, Analyse der Chroniktexte, (Mitteilungen der Antiquarischen Gesellschaft in Zürich, 52/2, 1984).

Haberkern P., After me There Will Come Braver Men': Jan Hus and Reformation Polemics in the 1530s, "German History”, 27 (2009), S. 177-195.

Happes J., Transformation und Nutzung der Konstanzer Konzilschronik im späten 15. Jahrhundert, „Mitteilungen der Residenzen-Kommission der Akademie der Wissenschaften zu Göttingen. Stadt und Hof", 4 (2015), S. 63-75.

Herkommer H., Die Geschichte vom Leiden und Sterben des Jan Hus als Ereignis und Erzählung, in: Literatur und Laienbildung im Spätmittelalter und in der Reformationszeit: Symposion Wolfenbüttel 1981, hg. v. L. Grenzmann, K. Stackmann, (Germanistische Symposien, Berichtsbände 5, 1984), S. 114-146.

Hoke R., Der Prozeß gegen Jan Hus und das Geleit König Sigmunds. Ein Beitrag zur Frage nach der Kläger- und Angeklagtenrolle im Konstanzer Prozeß von 1414/1415, „Annuarium Historiae Conciliorum“, 15 (1983), S. 172-193.

Hruschka C., Kriegsführung und Geschichtsschreibung im Spätmittelalter: eine Untersuchung zur Chronistik der Konzilszeit, (Kollektive Einstellungen und sozialer Wandel im Mittelalter, n.F. 5, 2001), S. 112-142.

Jost K., Konrad Justinger (ca. 1365-1438): Chronist und Finanzmann in Berns großer Zeit, (Vorträge und Forschungen, Sonderband 56, 2011).

Kautzsch R., Die Handschriften von Ulrich Richentals Chronik des Konstanzer Konzils, „Zeitschrift für die Geschichte des Oberrheins“, 9 (1894), S. 443-496, Tafel XVII-XXI.

Kejř J., Die Causa Johannes Hus und das Prozessrecht der Kirche, (2005).

Kubíková M., The heretic's Cap of Jan Hus, „Bohemian Reformation and Religious Praxis", 4 (2002), S. 143-150.

Löther A., Rituale im Bild. Prozessionsdarstellungen bei Albrecht Dürer, Gentile Bellini und in der Konzilschronik Ulrich Richentals, in: Mundus in imagine: Bildersprache und Lebenswelten im Mittelalter: Festgabe für Klaus Schreiner, hg. v. ders., (1996), S. 99-123.

Die hussitische Revolution: religiöse, politische und regionale Aspekte, hg. v. F. Machilek, (Forschungen und Quellen zur Kirchen- und Kulturgeschichte Ostdeutschlands 44, 2012).

Matouš F., Johannes Hus in der Schweizer Chronistik des 15. und 16. Jahrhunderts, in: Jan Hus: zwischen Zeiten, Völkern, Konfessionen: Vorträge des internationalen Symposions in Bayreuth vom 22. bis 26. September 1993, hg. v. F. Seibt, (Veröffentlichungen des Collegium Carolinum 85, 1997), S. 367-373.

Matthiessen W., Ulrich Richentals Chronik des Konstanzer Konzils. Studien zur Be- 
handlung eines universalen Großereignisses durch die bürgerliche Chronistik, „Annuarium Historiae Conciliorum“, 17 (1985), S. 71-191, 323-455.

Maurer H., Schweizer und Schwaben: ihre Begegnung und ihr Auseinanderleben am Bodensee im Spätmittelalter, (Konstanzer Universitätsreden 136, $\left.{ }^{2} 1991\right)$.

Maurer H., Vom Konzil bis zum Beginn des 16. Jahrhunderts, (Konstanz im Mittelalter 2, 1989).

Mertens M., Früher Buchdruck und Historiographie. Zur Rezeption historiographischer Literatur im Bürgertum des deutschen Spätmittelalters beim Übergang vom Schreiben zum Drucken, in: Studien zum städtischen Bildungswesen des späten Mittelalters und der frühen Neuzeit: Bericht über Kolloquien der Kommission zur Erforschung der Kultur des Spätmittelalters, 1978 bis 1981, hg. v. B. Moeller (et. al.), (Abhandlungen der Akademie der Wissenschaften in Göttingen, PhilologischHistorische Klasse 3/137, 1983), S. 83-111.

Die Appenzellerkriege - eine Krisenzeit am Bodensee, hg. v. P. Niederhäuser, (Forschungen zur Geschichte des Vorarlbergs 7, 2006).

Katalog der deutschsprachigen illustrierten Handschriften des Mittelalters 3, hg. v. N. H. Ott (et. al.), (2011), S. 450-487.

Ott N. H., Zum Ausstattungsanspruch illustrierter Städtechroniken. Sigismund Meisterlin und die Schweizer Chronistik als Beispiele, in: Poesis et pictura: Studien zum Verhältnis von Text und Bild in Handschriften und alten Drucken; Festschrift für Dieter Wuttke zum 60. Geburtstag, hg. v. S. Füssel, J. Knape, (Saecula spiritalia, Sonderband, 1989), S. 77-106.

Randák J., Jan Hus 600 - ein unübersichtliches Jubiläum, 2016, abrufbar im Internet: http://erinnerung.hypotheses.org/583 (besucht am 14.4.2016).

Riegel J., Die Teilnehmerlisten des Konstanzer Konzils. Ein Beitrag zur mittelalterlichen Statistik, (1913).

Rolker C., Die Richental-Chronik als Wappenbuch, „Deutsches Archiv für die Erforschung des Mittelalters“, 71 (2015), S. 57-103.

Roth E., Der volkreiche Kalvarienberg in Literatur und Bildkunst des Spätmittelalters, (Philologische Studien und Quellen 2, ${ }^{2} 1967$ ).

Rychterova P., Die Verbrennung von Johannes Hus als europäisches Ereignis. Öffentlichkeit und Öffentlichkeiten am Vorabend der hussitischen Revolution, in: Politische Öffentlichkeit im Spätmittelalter, hg. v. M. Kintzinger, B. Schneidmüller, (Vorträge und Forschungen 75, 2011), S. 361-382.

Saurma-Jeltsch L. E., Die Illustrationen und ihr stilistisches Umfeld, in: Diebold Schillings Spiezer Bilderchronik, Kommentar zur Faksimileausgabe der Handschrift Mss. h. h. I. 16 der Burgerbibliothek Bern, hg. v. H. Haeberli, C. v. Steiger, (1990), S. 31-71. 
Schenk G. J., Die Lesbarkeit von Zeichen der Macht und die Grenzen der Macht von Zeichen auf dem Konstanzer Konzil am Beispiel des Einzugs Papst Johannes'XXIII. (1414), in: Das Konstanzer Konzil als europäisches Ereignis: Begegnungen, Medien und Rituale, hg. v. B. Studt, G. Signori, (Vorträge und Forschungen 79, 2014), S. 255-304.

Schmid-Keeling R., Die Stadtansichten in den Schweizer Bilderchroniken, in: Schweizer Städtebilder. Urbane Ikonographien (15.-20. Jahrhundert), hg. v. B. Roeck (et al.), (2013), S. 37-49.

Das Konstanzer Konzil als europäisches Ereignis: Begegnungen, Medien und Rituale, hg. v. G. Signori, B. Studt, (Vorträge und Forschungen 79, 2014).

Šmahel F., Die hussitische Revolution, 3 Bde., (Monumenta Germaniae HistoricaSchriften 43, 2002)

Spiegel G. M., History, Historicism, and the Social Logic of the Text in the Middle Ages, „Speculum“, 65 (1990), S. 59-86.

Stettler B., Die Eidgenossenschaft im 15. Jahrhundert. Die Suche nach einem gemeinsamen Nenner, (2004).

Strahm H., Der Chronist Konrad Justinger und seine Berner Chronik von 1420, (Schriften der Berner Burgerbibliothek, 1978).

Studt B., Zwischen Kurfürsten, Kurie und Konzil. Die Hussitenpolitik König Sigismunds, in: Sigismund von Luxemburg: ein Kaiser in Europa: Tagungsband des internationalen historischen und kunsthistorischen Kongresses in Luxemburg, 8.-10. Juni 2005, hg. v. M. Pauli, F. Reinert, (2006), S. 13-23.

Philologie als Textwissenschaft. Alte und neue Horizonte, hg. v. H. Tervooren, H. Wenzel, (Zeitschrift für Deutsche Philologie, Sonderheft 116, 1997).

Urbach D., Weltgericht und städtische Selbstdarstellung. Das Wandgemälde am Triumphbogen des Ulmer Münsters, (2001), abrufbar im Internet: https://www.freidok.uni-freiburg.de/fedora/objects/freidok:267/datastreams/FILE1/con-tent (besucht am 17.10.2015).

Vorgrimler H., Die Geschichte der Hölle, (1994), abrufbar im Internet: http://digi20. digitale-sammlungen.de/de/fs1/object/display/bsb00041272_00001.html (besucht am 17.10.2015).

Wacker G., Ulrich Richentals Chronik des Konstanzer Konzils und ihre Funktionalisierung im 15. und 16. Jahrhundert. Aspekte zur Rekonstruktion der Urschrift und zu den Wirkungsabsichten der überlieferten Handschriften und Drucke, (2002), abrufbar im Internet: https://publikationen.uni-tuebingen.de/xmlui/handle/10900/46177 (besucht am 21.10.2015). 
